\title{
Delays in the referral and treatment of oral squamous cell carcinoma
}

\author{
P. Hollows, ${ }^{1}$ P. G. McAndrew, ${ }^{2}$ and M. G. Perini, ${ }^{3}$
}

Objective To investigate the delays in referral and treatment of patients with oral cancer.

Design A retrospective study.

Setting District General Hospital Maxillofacial Unit (MFU). Subjects 100 consecutive patients with invasive squamous cell carcinoma of the oral cavity referred to Rotherham District General Hospital Maxillofacial Unit (RDGH MFU) between 15th March 1993 and 16th January 1998.

Method Information collected at the time of referral and treatment was examined retrospectively.

Results In the patients studied $72 \%$ were male, mean age 61.2 years (sd $=11.2$, range 37 to 88 ) and $28 \%$ female, mean age 65.6 years ( $\mathrm{sd}=16.7$, range 29 to 90 ). The majority of referrals were from medical practitioners $(56 \%)$ and most of the remainder being referred by dental practitioners $(36 \%)$. The patient delay was found to be the most significant with only $39 \%$ presenting within 4 weeks, $29 \%$ delayed more than 3 months. There was no statistical correlation between T-stage, alcohol or cigarette use and the patient delay in presentation. Having presented to a medical or dental practitioner $69 \%$ were referred within 1 week. There were no significant differences between the T-stages presenting to either medical or dental practitioners or in their delay in referral for each stage. There was no significant difference in age or sex distribution between the populations presenting to general medical or general dental practitioners. General medical practitioners were more likely to refer a patient urgently. Patients referred directly to the MFU were seen quickly but those referred via an indirect route were delayed. $95 \%$ of patients were treated within 6 weeks of first consultation.

Conclusion The majority of practitioners refer patients with oral cancer within 1 week. The most significant delay is that caused by the patient. Some practitioners referred patients to inappropriate specialities, leading to indirect referrals. This results in additional delay in the referral and treatment pathway. Education of the public and primary health care workers should continue. Opportunistic screening of the oral mucosa should be part of the dental check up, with possible targeting of patients at greatest risk, particularly heavy drinkers and smokers.

$\mathrm{T}$ he British Dental Association has defined the term 'oral cancer' using the World Health Organisation's International Classification of Diseases (ICD), version 9. ${ }^{1}$ The sites included are the lip (code 140), tongue (141), gum (143), floor of mouth (144), other

${ }^{1}$ Senior Registrar, ${ }^{2}$ Consultant, Maxillofacial Unit, Rotherham District General Hospital, Moorgate Road, Oakwood, Rotherham, South Yorkshire S60 2UD;

${ }^{3}$ SHO, Maxillofacial Unit, Royal Preston Hospital, Green Lane, Preston,

Lancashire PR2 9HT

Correspondence to: Mr M. G. Perini, 334 Trowell Road, Nottingham NG8 2DT

email:mg.perini@telinco.co.uk

REFEREED PAPER

Received 09.10.98; accepted 30.11.99

(C) British Dental Journal 2000; 188: 262-265 unspecified parts of the mouth (145), oropharynx (146), hypopharynx (148) and other ill defined sites within the lip, oral cavity and pharynx (149). Using this definition there are currently 3,400 new cases of oral cancer a year and about 1,600 deaths with oral cancer accounting for $1 \%$ of all new cancer registrations. The overall 5-year survival for oral cancer in England and Wales is about $50 \%$, however, if detected early then it is anticipated that this could be improved to $80 \%{ }^{2}$ There have been great advances made in the management of this condition, from improved diagnostic imaging of the tumour to sophisticated reconstructive procedures including oral implantology to restore the dentition. Although the quality of life of these unfortunate patients has been improved, survival figures have not changed greatly.

Early detection of oral cancer is an important objective as it should improve outcome and reduce the morbidity of treatment. In order to do this it is necessary to raise awareness in primary healthcare workers, who are in a position to diagnose the condition and within the population as a whole. There have been many studies on delays in referral of patients with oral cancer. ${ }^{3-9}$ This study looks at the delays incurred in the referral and treatment of oral cancer to a single MFU in a district general hospital setting and attempts to elucidate factors that may increase the delay.

\section{Method}

One hundred consecutive cases of oral squamous cell carcinoma referred to RDGH MFU were studied between 15th March 1993 and 16th January 1998. Only histologically proven invasive squamous cell carcinomas were included. The patient's sex and age were noted, and the primary site and $\mathrm{T}$ stage defined at the time of the first visit. Details of the patient's alcohol and tobacco use were recorded. The patient's delay was defined as the time between the onset of symptoms to first appointment at a primary care facility. Some of the patients described a very long delay which could represent a possible pre-malignant condition or pre-existing oral condition. It is not possible to determine exactly when the malignant condition started for obvious reasons. Having been seen in the primary care setting the practitioner's delay was defined as the time from first visit to the date of referral. In some cases this could not be determined from the patient and therefore the practitioner was contacted and details from their records retrieved. Where the originating practitioner was neither a dental nor medical practitioner the delays were not included in the analysis. The urgency of each referral was determined. If a referral letter mentioned malignancy or the possibility of a tumour it was categorised as 'urgent'. Features such as long standing ulceration were designated 'suspicious', but if there was no indication of what the pathology may be it was interpreted as a 'routine' referral. Any accompanying telephone call immediately categorised a referral as 'urgent'. The delay to the MFU was defined as the time between the date of the letter or telephone call from the practitioner to first attendance at the MFU. Where delays could not be determined, cases were excluded. Some patients were not referred directly to our department and came via other routes. These were termed indirect referrals. Once the patients had been 


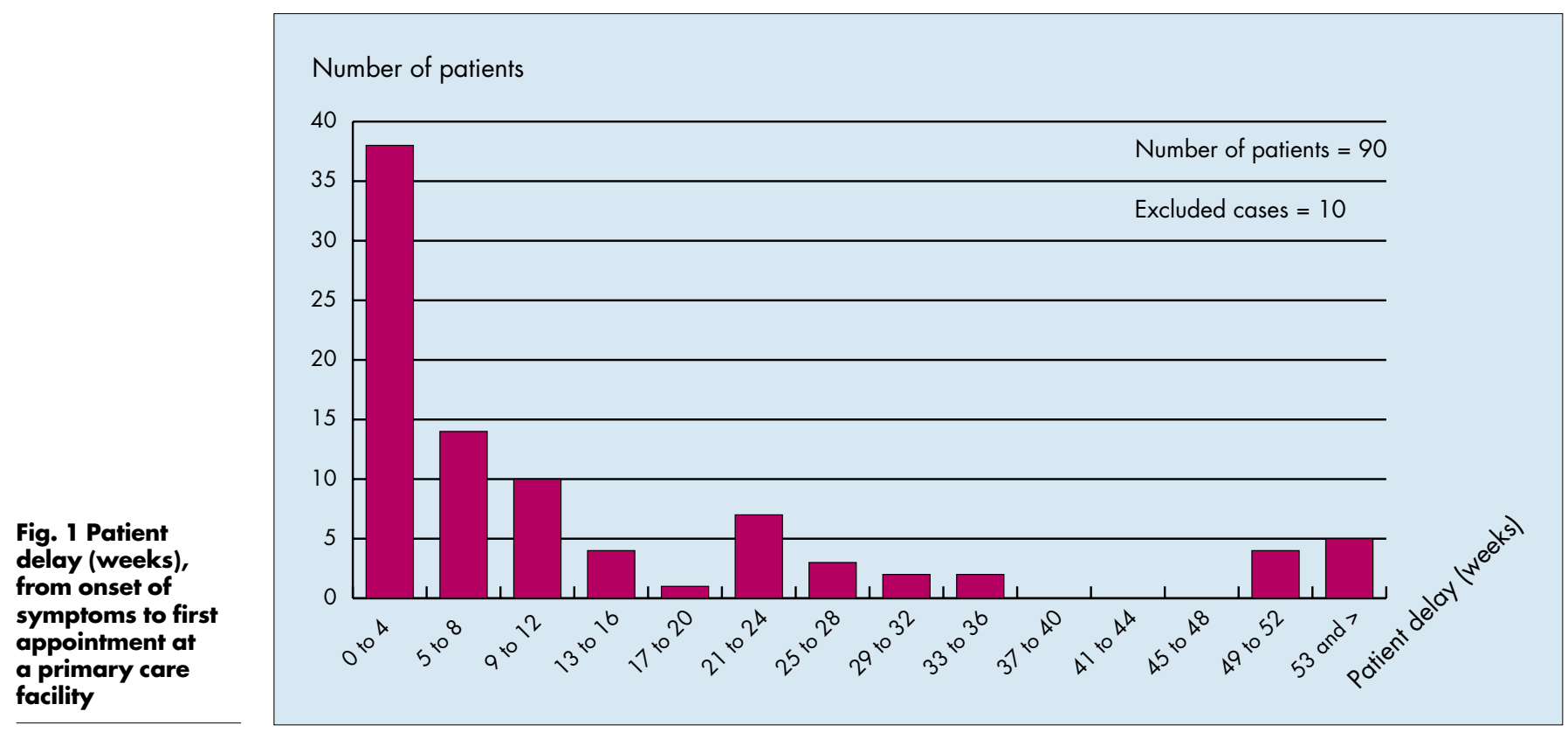

Fig. 1 Patient (n) symptoms to first facility seen in our department the delay to definitive treatment was noted. If the patient was referred for primary radiotherapy then the time to first appointment in the radiotherapy clinic was given.

\section{Results}

In our cohort of 100 patients with invasive oral squamous cell cancer $28 \%$ were female and $72 \%$ male. In males the mean age was 61.2 years ( $\mathrm{sd}=11.2$, range 37 to 88$)$ and females 65.6 years $(\mathrm{sd}=$ 16.7, range 29 to 90). As expected the commonest sites were the tongue ( $31 \%$ ) and the 'sump' areas of the oral cavity comprising the floor of mouth ( 19\% ), retromolar trigone (17\% ) and lower alveolus ( $16 \%$ ). Only $11 \%$ of patients did not smoke or drink alcohol, all of this group were female. $56 \%$ of patients were referred by general medical practitioners (GMPs) and $36 \%$ by general dental practitioners (GDPs). Two patients who attended the accident and emergency department had previously seen both medical and dental practitioners who had failed to diagnose extensive (T4) tumours.

Patient delay is shown in Figure 1. The mean delay was 22.5 weeks, sd $=62.1$ with only $39 \%$ of patients presenting within 4 weeks; $29 \%$ delayed more than 3 months. The mean patient delay presenting to GDPs was 20 weeks ( $s d=45.3$, range 0 to 260 ), for
GMPs this was 25 weeks ( $s d=74$, range 0 to 520 ). There was no correlation between patient delay in cigarette smokers and the quantity consumed (correlation coefficient $=0.12$ ). Similarly no correlation was found between patient delay and alcohol intake (correlation coefficient $=0.05$ ). Patient delay for each anatomical site is shown in Figure 2, however, because of small numbers, caution is necessary in interpreting this information.

There was no significant difference between the age $(\mathrm{t}=-0.1,82 \mathrm{df}$, $P=0.92)$, sex $\left(\chi^{2}=2.6,1 \mathrm{df}, \mathrm{p}=0.05\right)$ or T stage $\left(\chi^{2}=2.88,3 \mathrm{df}, P=\right.$ $0.05)$ distribution of patients attending GMPs or GDPs. Figure 3 shows practitioner delay from first consultation to referral. Sixty-nine per cent of patients were referred within 1 week. The mean delay in referral for GDPs was 14.5 days ( $\mathrm{sd}=32.3$, range 0 to 176 ) and for GMPs 8.4 days ( $s d=17.6,0$ to 90 ). Of the 92 referral letters sent by practitioners only $34 \%$ were interpreted as being urgent by the receiving consultant, $27 \%$ were referred by GMPs and $7 \%$ by GDPs. This difference was significant $\left(\chi^{2}=7.68,1 \mathrm{df}, P=0.05\right)$ with GMPs classifying a referral more appropriately. Of the patients seen at $\mathrm{RDGH}$ MFU, 54\% were direct referrals and $46 \%$ were indirect referrals. The time to the patient's first consultation at RDGH MFU was then assessed (Figure 4). Significant delay was incurred when patients were

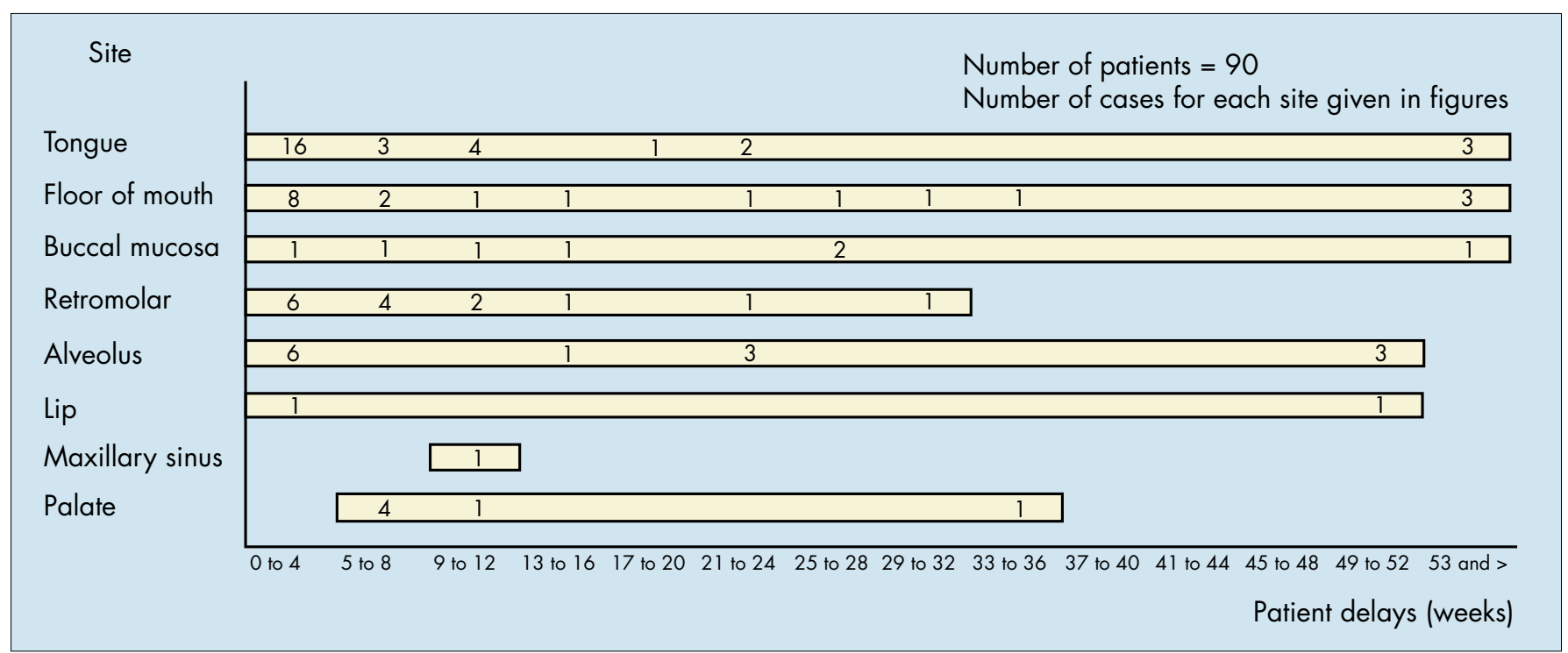

Fig. 2 Patient delay (weeks), from onset of symptoms to first appointment at a primary care facility versus site of SCC 
referred indirectly $(\mathrm{t}=2.56,89 \mathrm{df}, P=0.012)$. Having been seen at RDGH MFU the delay to definitive treatment is shown in Figure 5. Only five patients received treatment later than 6 weeks from presentation.

\section{Discussion}

In this group of patients the age range and sites for oral cancer were similar to previous studies. ${ }^{9,10}$ Worrall (1995) in his audit of oral squamous cell carcinoma found that the mean age of presentation occurred around 5 years earlier in men and our current study confirms this finding. ${ }^{11}$ (It is clear that as a group of patients' alcohol and tobacco use is common.) It is known that the use of alcohol is strongly associated with oral and pharyngeal cancers and that smoking concurrently multiplies the risk. Blot indicated that the relative risk for oral and pharyngeal cancer in heavy consumers of both products exceeds the risks for abstainers by 37 -fold. ${ }^{12}$ Both general dental and medical practitioners are well placed to help in the primary prevention of this disease and should take the opportunity to give advice on alcohol and tobacco consumption. Patients who fall into the high risk group for oral cancer using both tobacco and alcohol should ideally have oral mucosal screening. Large numbers of the population attend the dentist for regular check ups and opportunistic screening can easily be carried out in this group. It was shown by Cowan et al. in 1995 that $94 \%$ of dentists include soft tissue examination in their regular dental check up, but only $14 \%$ said their records contained information about alcohol and tobacco consumption. ${ }^{13}$ Field et al. showed that mucosal screening can be easily integrated into routine dental care. ${ }^{14}$ Studies that have looked at true screening programmes have met with limited success, essentially because of the low incidence of oral cancer in the population at large and poor compliance. ${ }^{15,16}$ One of the major factors in the lateness of diagnosis has been the patient's own delay in presenting after the onset of symptoms. Our study shows that a large number of patients are still presenting with advanced disease and only $39 \%$ of patients attended within 4 weeks of the onset of symptoms and 29\% after 3 months, which is similar to the findings of Dimitroulis et al. ${ }^{6}$ There are many factors for such delay including self treatment and fear of 'going to the dentist' but the mainstay of improving the situation must be patient education. The present study did not show any correlation between alcohol intake and amount smoked and patients' delay, suggesting that oral awareness is not directly related to quantities consumed.
Fig. 3 Practitioner delay (days), from first consultation to referral
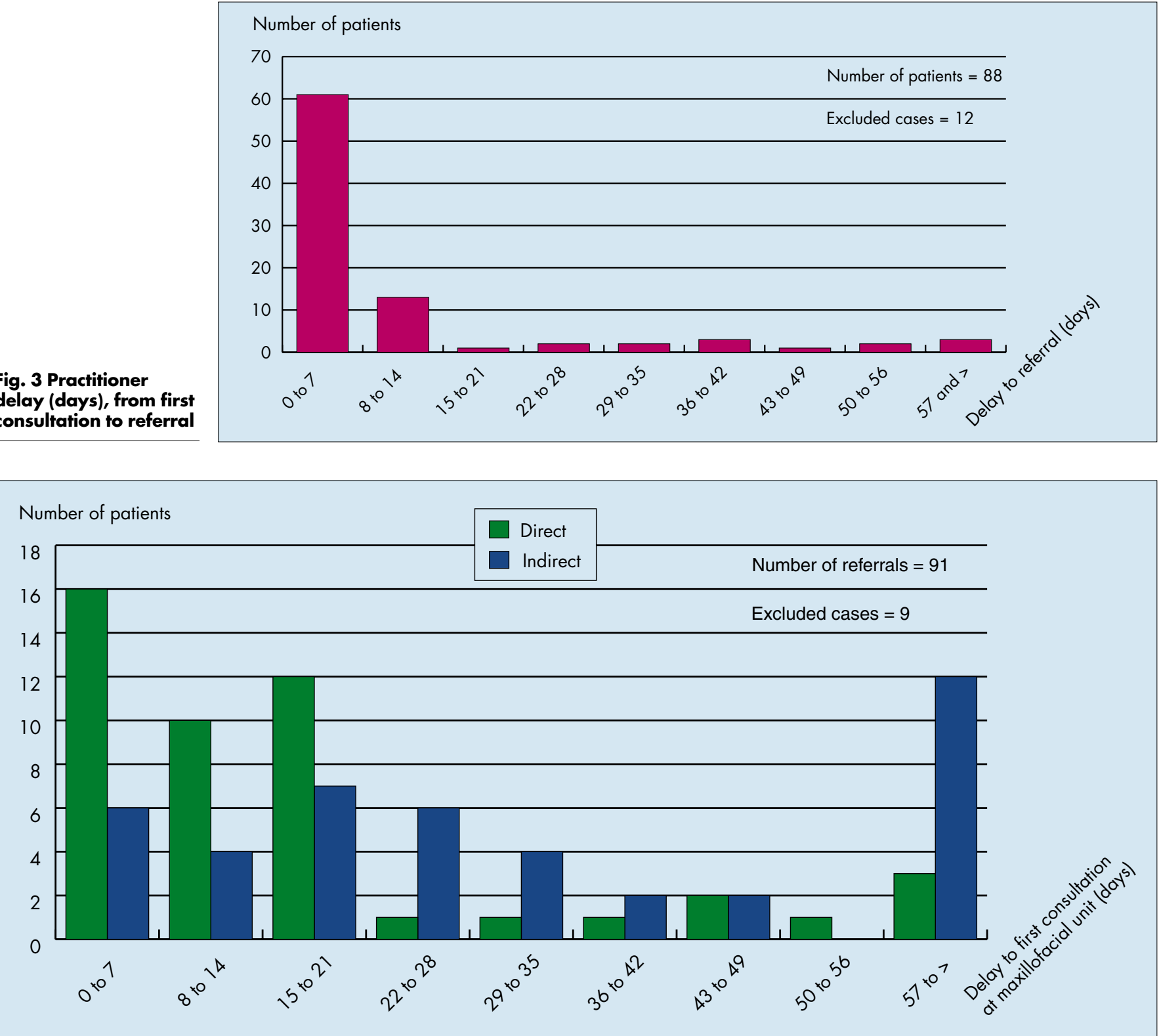

Fig. 4 Time taken from practitioner's initial referral to first consultation at RDGH Maxillofacial Department (days). A comparison between direct and indirect referral routes 


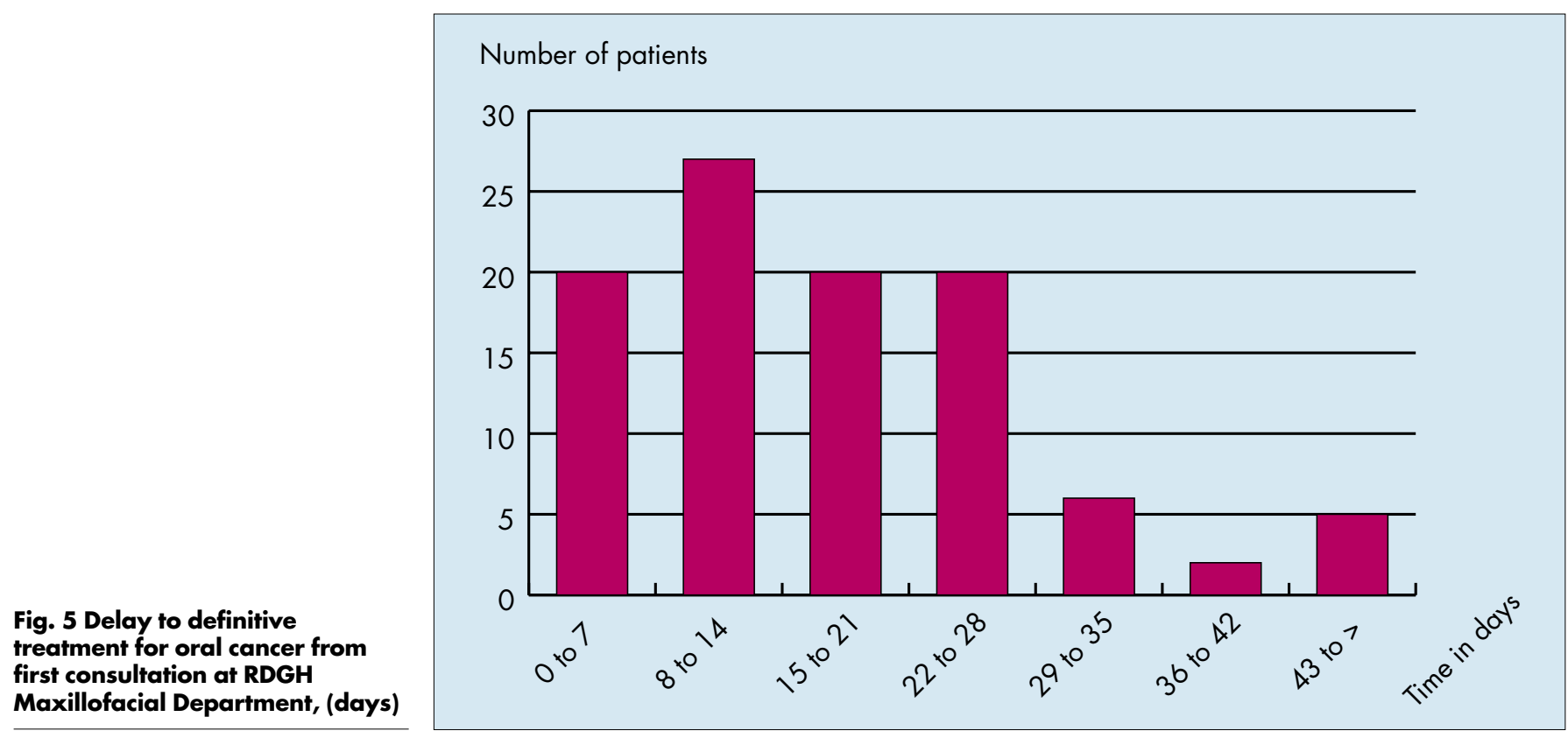

The delay associated with the primary care practitioner was not seen to be a significant problem in our study. The cohort of patients presenting to GDPs and GMPs were similar for sex, age and T stage distributions. This is in contrast to other papers that indicated that GMPs saw more advanced lesions and older patients. ${ }^{5,6}$ Two patients did however get referred from the accident and emergency department having attended in pain. In both of these cases the dental and medical practitioners had examined them in the previous 6 weeks but failed to diagnose the condition. These two cases highlight a small minority of practitioners who have difficulty in diagnosing serious mucosal pathology. Some delay could be avoided if practitioners described more accurately their clinical findings, and stated clearly that they thought the patient in question may have a malignancy. When the referral letters were classified in degrees of urgency it was surprising to find that only $34 \%$ were referred as urgent. GMPs were more likely to refer patients urgently. This has a direct bearing on how soon the patient is seen in outpatient clinics. It can be difficult for the consultant in charge to choose who should be seen as a priority from the information given in the referral letter. Only a small number of practitioners (11\%) telephoned to discuss the case. Medical and dental schools should give consideration to improving the skills of undergraduates in patient referral and letter writing. Only $50 \%$ of the patients referred to our department arrived directly from the primary care setting. It was clear from our results that direct referrals were seen more quickly. Practitioners need to be informed who is the most appropriate specialist to refer suspected oral cancer to in their locality. Having been seen by a specialist the delay to treatment was minimal with only five patients waiting more than 6 weeks for treatment. The authors feel that this period of delay is acceptable as these patients require complex investigations and diagnostic imaging prior to definitive treatment. Some time is also needed for counselling and support by the multidisciplinary team managing this condition

\section{Conclusion}

Delay in the diagnosis of oral cancer is caused by many factors. Patient delay is usually the most significant factor. Most general dental and medical practitioners recognise oral cancer and refer early. A small number however, continue to misdiagnose and inappropriately treat cases. Education of both patients and practitioners would still seem to be appropriate. The majority of dental practitioners screen the oral mucosa as part of their dental check up and it could be argued that these should be free of charge to encourage attendance. Medical practitioners should take the opportunity to screen patients, particularly those at high risk using tobacco and alcohol. When referring patients the practitioner should be clear who actually treats the condition as indirect referrals invariably lead to delay. Practitioners should be encouraged to telephone departments directly to discuss cases and referral letters should clearly indicate suspicions of malignancy.

1 International Classification of Diseases, Injuries and Causes of Death, Ninth Revision. Geneva: WHO, 1977.

2 An Oral Health Strategy for England and Wales. London. Department of Health, 1994.

3 Shafer G W. Initial management and delay in the diagnosis of oral cancer. $J$ Am Dent Assoc 1975; 90: 1262-1264.

4 Cooke B E D, Tapper-Jones L. Recognition of oral cancer, causes of delay. $\mathrm{Br}$ Dent J 1977; 142: 96-98.

5 Scully C, Malamos D, Levers B G H , Porter S R,Prime S R. Sources and patterns of referrals of oral cancer: role of general practitioners. $\mathrm{Br}$ Med J 1986; 293: 599-602.

6 Dimitoulis P R,Wiesenfield D. Referral patterns of patients with oral squamous cell carcinoma, Australia. Oral Oncol, Eur J Cancer 1992; 28B: 23-27.

7 Jovanovic A, Kostense P J, Schulten E A J M, Snow G B, Van der Waal I. Delay in the diagnosis of oral squamous cell carcinoma; A report from the Netherlands. Oral Oncol, Eur J Cancer 1992; 28B: 37-38.

8 Kowalski L P, Franco E L, Torloni H et al. Lateness in diagnosis of oral and oropharyngeal carcinoma: Factors related to the tumour, the patient and health professionals. Oral Oncol, Eur J Cancer 1994; 30B: 167-173.

9 Pinholt E M, Rindum J, Pindborg J J. Oral cancer: a retrospective study of 100 Danish cases. Br J Oral Maxillofac Surg 1997; 35: 77-80.

10 Schnetler J F C. Oral cancer diagnosis and delays in referral. Br J Oral Maxillofac Surg 1992; 30: 210-213

11 Worrall S F, Corrigan M. An audit of one surgeon's experience of oral squamous cell carcinoma using a computerised malignancy database. Ann R Coll Surg Engl 1995; 77: 332-336.

12 Blot W J. Alcohol and Cancer. Cancer Res (Suppl.) April 1992; 52: 2119s2123s.

13 Cowan C G, Gregg T A, Kee F. Prevention and detection of oral cancer: the views of primary care dentists in Northern Ireland. Br Dent J 1995; 179: 338-342.

14 Field E A, Morrison T, Darling A E, Parr T A, Zakrzewska J M. Oral mucosal screening as an integral part of routine dental care. Br Dent $J 1995$; 179: 262-266.

15 Jullien J A, Zakrzewska J M, Downer M C, Speight P M. Attendance and compliance at an oral cancer screening programme in a general medical practice. Oral Oncol, Eur J Cancer 1995; 31B: 202-206.

16 Ikeda N, Downer M C, Ishii T, Fukano H, Nagao T, Inoue K. Annual screening for oral cancer and precancer by invitation to 60-year-old residents of a city in Japan. Comm Dent Health 1995; 12: 133-137. 